\title{
Individual versus social housing during quarantine
}

As most people in laboratory animal science know, environmental enrichment is used to promote the psychological well-being of the animals under our care. Along this line, the Guide for the Care and Use of Laboratory Animals highlights the importance of social housing for normally social species except when experimental requirements or veterinary concerns intervene ${ }^{1}$. Nevertheless, Dr. Alison Wright, a relatively new user of rhesus monkeys, believed that social housing could, at times, be more detrimental than helpful for her animals. "Listen to me," said Wright to Larry Covelli, the chairman of the Great Eastern University IACUC, "nobody wants unstressed animals more than I do. Stressed monkeys would probably affect my research results. But it seems to me that if we keep them together in the same pairs that the vendor set up while they're in their quarantine period, and then afterwards I house them individually because of the research I do, they're going to be more stressed than if I housed them individually to begin with and gave them a chance to adapt to that condition. Doesn't that make sense to you?"

Covelli could see the question coming, and he wished he had the Attending Veterinarian (AV) and the entire IACUC by his side. "Look, I know you're upset, Alison, but the animals are going to be in quarantine for at least six weeks, maybe longer, and that in itself is going to be a stressor even with pair housing. The IACUC agreed with you that individual housing is needed for your research, but the IACUC and the AV also are in agreement it will be less of a stress on the animals to house them in pairs now, let them adapt to their new environment, and once your experiments begin, keep them close to each other, in adjoining cages, so that they can see and smell each other and even have finger contact. Not only do we think that will be less stressful, but it will also meet federal regulations for social housing whenever possible. Overall, we think this is the best way to do things for the animals and for your research."

What do you think? Will separating the monkeys after the quarantine period is complete be less or more stressful for them as compared with separating them early in the quarantine period as the investigator has requested?

1. Institute for Laboratory Animal Research. Guide for the Care and Use of Laboratory Animals 8th edn. (National Academies Press, Washington, DC, 2011).

\section{RESPONSE}

\section{Single housing should be minimized}

Mary Ellen Goldberg, BS, VMT, LVT, CVT, SRA

I have seen both strategies used. I believe that because the vendor is sending the primates already housed in pairs, they should be allowed to acclimate to their new home without disrupting this order. The travel will be enough of a stress to the monkeys.

I am completely sympathetic with the Principal Investigator wanting to begin her research as quickly as possible without interference from behavioral issues. But the Guide for the Care and Use of Laboratory Animals ${ }^{1}$ clearly states, and other guidelines also recommend ${ }^{2}$, that single housing of social animals should be kept to a minimum period. I feel that the monkeys should remain in pairs during the quarantine period to help mitigate stress ${ }^{2-4}$.
Once the quarantine period is over, the primates can be housed individually in adjoining cages, with visual, olfactory and finger contact ${ }^{1,5}$, possibly allowing them to groom each other.

Exceptional scientific or veterinary justification is required for single housing of primates. Such justification could include research-related exemptions (viral or antibody status, requirement for continuous monitoring or sample collection, or special instrumentation) or veterinary-related concerns (vicious or overly aggressive behavior, debilitation as a result of age or other conditions or presence of contagious disease).

1. Institute for Laboratory Animal Research. Guide for the Care and Use of Laboratory Animals 8th edn. 69 (National Research Council, Washington, DC, 2011)

2. National Centre for the Replacement, Refinement and Reduction of Animals in Research. NC3Rs Guidelines: Primate Accommodation, Care and Use (National Centre for the Replacement, Refinement and Reduction of Animals in Research, London, 2006). <http:// www.nc3rs.org.uk/downloaddoc.asp?id=418>

3. Baumans, V. et al. Making use of the stress buffering influence of a companion. in Making Lives Easier for Animals in Research Labs: Discussion by the Laboratory Animal Refinement \& Enrichment Forum 101 (Animal Welfare Institute, Washington, DC, 2007).

4. Reinhardt, V. Comfortable quarters for primates in research institutions. in Comfortable Quarters for Laboratory Animals 9th edn. 65 (Animal Welfare Institute, Washington, DC, 2002).

5. Fortman, J.D., Hewett, T.A. \& Bennett, B.T. Husbandry. in The Laboratory Nonhuman Primate 47, 49 (CRC Press, Boca Raton, FL, 2002).

Goldberg is Instructor at VetMedteam, LLC.

\section{RESPONSE}

\section{Solid advice from the IACUC chair}

James F. Taylor, DVM, MS, DACLAM

Although the scenario does not provide the justification for individual housing 